\title{
Vigilância epidemiológica no plano de intensificação das ações de controle de malária no estado de M ato Grosso: estudo de caso
}

\author{
Epidemiologic surveillance in the plan for intensification of \\ malaria control actions in the state of M ato Grosso: a case study
}

N oemi Dreyer Galvão ${ }^{1}$

Edna M assae Yokoo ${ }^{2}$

M arina Atanaka dos Santos ${ }^{3}$

Sonia Natal 4,5

\footnotetext{
${ }^{1}$ Coordenadoriade Vigilância Epidemiológica, Secretaria de Estado de Saúde de M ato Grosso. Rua 07, Quadra 16, Bloco 05, Centro Político Administrativo, Palácio Paiaguas. 78050-970 Cuiabá MT.

noemidgalvao.mt@terra.com.br

${ }^{2}$ Departamento de

Epidemiologia e

Bioestatística, Universidade

Federal Fluminense.

${ }^{3}$ Instituto deSaúde

Coletiva, Universidade

Federal de M ato Grosso.

${ }^{4}$ Departamento de

Endemias Samuel Pessoa,

Escola Nacional deSaúde

Pública, Fiocruz.

${ }^{5}$ Grupo deEstudos de

Gestão eAvaliação, Instituto

$M$ aterno Infantil Professor

Fernando Figueira.
}

Abstract The objective of this study is to analyze theepidemiologic surveillance in the M alaria Control Actions Intensification Plan (PIACM) in the 18 cities of the state of M ato Grosso, from 1999 to 2002. An evaluative research of the type implantation analysis was conducted. The analysis was carried out using a logic model considering 4 dimensions - external and political context, organizational context, implementation and effectiveness - and 5 evidence bases, 3 of primary data and 2 of secondary data. The cities are called CASE. The data were collected using semi-structured questionnaires. Theimplementation of thePIACM actions was found adequate in only $5,6 \%$ of CASES. The effectiveness of the actions was adequate in only one CASE and none of the CASES showed adequate contextual characteristics. The principal factors affecting the implementation were: fre quent changes of the local health managers and professionals, fragmented and sporadic training and health promotion and prevention actions limited to the primary careteam. In conclusion, structural problems and lack of articulation of epidemiologic surveillancehamper the full development of the PIACM actions.

Key words M alaria, Epidemiologic surveillance, Health services evaluation, PIACM
Resumo Esteestudo tem como objetivo analisar a vigilância epidemiológica no Plano de Intensificação das A ções de Controle de M alária (PIACM ), nos dezoito municípios mato-grossenses, de 1999 a 2002. Realizou-seuma pesquisa avaliativa do tipo análisedeimplantação. A análisefoi realizada através do M odelo Lógico de Avaliação, considerando quatro dimensões de análise - contexto externo e político, contexto organizacional, implementação e efetividade - e cinco bases de evidências, sendo três de dados primários eos outros secundários. Os municípios foram denominados de CASOS. Os dados foram coletados por questionários semi-estruturados. A implementação das ações do PIACM apresentou-se adequada apenas em $5,6 \%$ dos CASOS. A efetividade das ações em apenasum CASO e nenhum CASO teve condição adequada nas características contextuais. 0 s fatores que mais influenciaram a implementação foram: rotatividade dos gestoresmunicipais e profissionais de saúde; capacitação fragmentada e esporádica; promoção e prevenção à saúde limitada aos profissionais da atenção básica. Conclui-se que a vigilância epidemiológica, no PIACM, mostrou-se desarticulada, com problemas de ordem estrutural, o que vem dificultando o pleno desenvolvimento das ações. Palavras-chave M alária, Vigilância epidemiológica, Avaliação de programas de saúde, PIACM 
Introdução

A ocorrência demalária no mundo aponta a persistência da doença, principal menteem países das regiões tropical e sub-tropical, que registram anualmente de 300 a 500 milhões de casos novos e mais de um milhão de mortes ${ }^{1}$.

No Brasil, em 2000, a Amazônia Legal concentrou cerca de $99,7 \%$ dos casos registrados e dela foi provenientea maioria dos casos detectados em outros estados do país ${ }^{2,3,4}$. A pesar disso, reduziu-se a incidência na região amazônica de 31,9 (1999) para 15,9 por 1.000 habitantes $(2002)^{5}$. Em 2002, foram identificados 72 municípios da região amazônica com alto risco para transmissão de malária, ou seja, com Incidência Parasitária Anual (IPA) igual ou maior que 50,0 casos por 1.000 habitantes 5 .

A persistência da malária na Amazônia Legal é determinada por diversos fatores, como pe quena e dispersa população; falta de saneamento básico, de pessoal capacitado e disponível, de continuidadeoperacional de estratégias deintervenção, de transporte ágil e, muitas vezes, de insumos básicos. Esses fatores, aliados às dificuldades político-administrativas de cada município, acabam por dificultar o controle da doença ${ }^{6}$.

Vários esforços foram realizados para o controle da malária ao longo de décadas, no Brasil, que resultaram na redução da morbidade e mortalidade a um padrão que não interfere na qualidade de vida das comunidades ${ }^{7,8}$. No entanto, a el evada incidência da doença na região amazôni$\mathrm{ca}$, devida à complexidadeecológica e social, desafia técnicos e governos. Os problemas operacionais dos sistemas locais de saúde, que afetam diretamente a vigilância da malária ${ }^{9}$, indicaram ao setor saúde a necessidade de implementação de novas estratégias de ação, especialmente as que propõem maior integração entre as áreas de prevenção/controle ea rede assistencial ${ }^{2}$.

Nesse sentido, a descentralização das ações de epidemiologia e controle de doenças para os estados e municípios foi um dos componentes da política de saúde no Brasil para implementar a integração das ações de saúde. 0 processo de descentralização do controle da malária foi incrementado com a implantação do Plano de Intensificação das Ações de Controle da M alária na Amazônia Legal (PIACM ) ${ }^{5}$, em 2000.

O PIACM foi lançado pelo M inistério daSaúde (M S), em parceria com estados e municípios, a fim de reduzir a metade da incidência da malária na região amazônica. 0 plano estava alicerçado nos componentes de vigilância epidemiológi- ca (VE), assistência ao paciente, promoção e mobilização social. As suas estratégias de intervenção foram implementadas e/ou fortalecidas de forma integrada, de acordo com as características da malária de cada localidade ${ }^{8}$. Assim, os estados e municípios assumiram a responsabilidade na organização e gestão das ações de controle da malária, revelando experiências diferenciadas no controledessa doença. 0 plano estabeleceu como prioridade 254 municípios da Amazônia Legal, dos quais vinte localizados no estado de Mato Grosso 5 .

O objetivo deste estudo foi avaliar a implementação do PIACM, com ênfase no componentevigilância epidemiológica, em dezoito municípios mato-grossenses.

\section{M etodologia}

\section{Desenho do estudo}

Para a análise de implantação, optou-se pelo estudo de casos múltiplos imbricados ${ }^{10-13}$, o qual oferece a vantagem de uma observação intensiva e particularizada da implantação.

Destaca-se, para análise deimplementação do PIACM /M T, o componente vigilância epidemiológica no controle da malária, em dezoito municípios do estado de Mato Grosso. 0 modelo lógico do programa ${ }^{12}$ e o de avaliação do PIACM , construído para este estudo, foram adaptados do modelo térico do Programa de Controle de Tuberculose ${ }^{12}$ (Figura 1).

Foram incluídos no Modelo Lógico de Avaliação (M LA) os componentes do PIACM : vigilância epidemiológica eambi ental, assistência ao paciente e promoção e mobilização social. Além disso, foram considerados o contexto externo e político, o contexto organizacional, a implementação do PIACM ea efetividade das ações de controle da malária. Estas quatro dimensões se subdividiram em subdimensões, de acordo com os fatores que podem influenciar positiva ou negativamentea intervenção do PIACM (Quadro 1).

A dimensão contexto externo e político foi subdividida em três subdimensões: condições socioeconômicas eambientais; projeto degoverno e governabilidade e capacidade de governo. Estas buscaram evidenciar, respectivamente: as condições sociais e econômicas do município; 0 reconhecimento político do gestor e o conhecimento do recurso financeiro; a equipe técnica, a intersetorialidade e a autonomia do gestor.

A dimensão contexto organizacional buscou 
identificar o planejamento e monitoramento das ações de vigilância epidemiológica da malária; a capacitação de recursos humanos; informação, educação e comunicação (IEC); mobilização social e satisfação do profissional de saúde.

A dimensão implementação foi subdividida em três subdimensões: integralidade e qualidade da vigilância epidemiológica e ambiental; integralidade equalidade dos serviços de saúde e implementação da infra-estrutura.

Finalmente, a dimensão efetividade foi trabal hada em três subdimensões: controle de risco para malária; controle de danos e satisfação do usuário.

As dimensões foram desagregadas em subdimensões e essas em indicadores e ou critérios, quantitativos equal itativos respectivamente. Para cada indicador, foi definido a fonte de evidência para a coleta de dados (Quadro 1).

Foi utilizado o modelo de avaliação rápida (REM $)^{14,15}$ e as bases de evidências incluídas foram: entrevista com o gestor, usuário e profissionais de saúde do município; dados dos sistemas de informação (SISM AL, SI M eSIH); dados do Departamento de Informação e Informática do Sistema Ú nico de Saúde (DATASUS), do Instituto Brasileiro deGeografia eEstatística(IBGE), e do Instituto $N$ acional de Colonização e Reforma Agrária (INCRA).

A matriz de julgamento foi baseada no M LA (Figura 2) e, ao conjunto de critérios eindicadores, foram atribuídos valores (escores) com a pontuação máxima do valor esperado. Os valores foram estabelecidos com base na literatura, em normas do M S, e na pactuação entre profissionais da SES/MT e DENSP/ENSP/FIOCRUZ e ISC/UFMT.

\section{Municípios do estudo}

Dos vinte municípios mato-grossenses previamente selecionados pelo PIACM ${ }^{16}$, o estudo englobou dezoito municípios edois foram excluídos devido a problemas de operacionalização e de recursos financeiros. Os municípios selecionados foram denominados de CASO e enumerados de 1 a 18, por ordem alfabética.

Os CASOS foram criados, em sua maioria, na década de 1980 e 1990, exceto os CASOS 2 e CASO 17, instituídos em 1943 e 1746, respectivamente. Esses dezoito municípios estão localizados, em média, a 774,5 quilômetros da capital do estado, variando mais ou menos de 276,08 quilômetros, e ainda apresentam grandes extensões territoriais com baixa densidade demográfica.

Quadro 1. Dimensões utilizadas para construção do MLA.

\begin{tabular}{|l|l|}
\hline \multicolumn{1}{|c|}{ Dimensões } & \multicolumn{1}{c|}{ Subdimensões } \\
\hline Contexto externo e político & $\begin{array}{l}\text { Condições socioeconômicas e ambientais; } \\
\text { Projeto de governo e governabilidade; } \\
\text { Capacidade de governo. }\end{array}$ \\
\hline Contexto organizacional & $\begin{array}{l}\text { Planejamento e monitoramento; } \\
\text { Capacitação de Recursos Humanos; } \\
\text { Opinião do RH; } \\
\text { Informação, educação e comunicação (IEC) e mobilização social. }\end{array}$ \\
\hline Implementação das ações de \\
controle da malária & $\begin{array}{l}\text { Integralidade e qualidade da vigilância epidemiológica e ambiental; } \\
\text { Integralidade e qualidade dos serviços de saúde; } \\
\text { Implementação da infra- estrutura. }\end{array}$ \\
\hline Efetividade das ações de controle \\
da malária & $\begin{array}{l}\text { Controle de riscos; } \\
\text { Controle de danos; } \\
\text { Satisfação dos usuários. }\end{array}$ \\
\hline
\end{tabular}

Fonte: Adaptado deN atal et al. ${ }^{12}$. 
Componente inovador do PIACM - financiamento das ações de epidemiologia e controle de doenças.
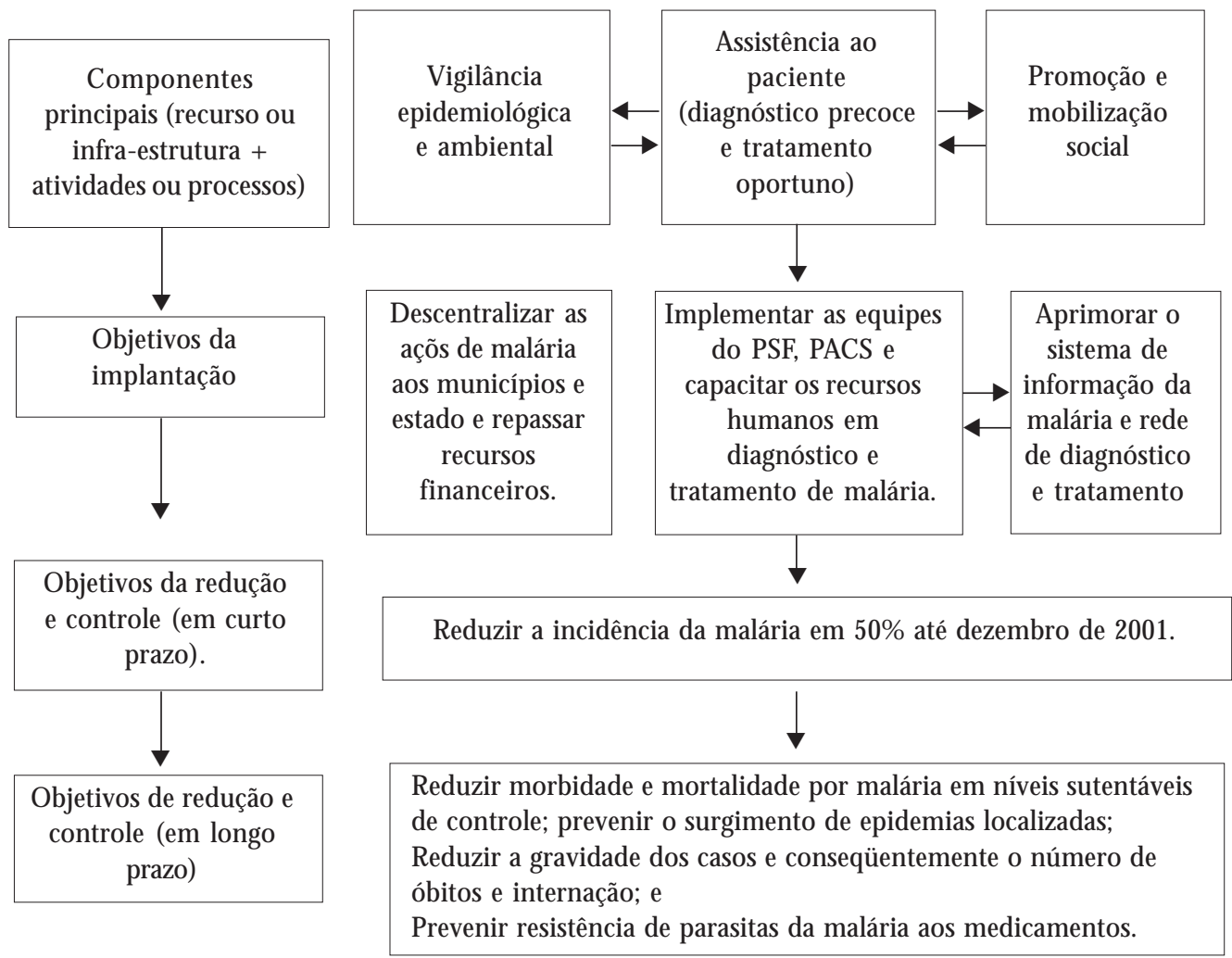

Fonte: Adaptado deN atal et al. ${ }^{12}$

Figura 1. Modelo lógico do PIACM.

\section{Unidades de análise}

As Secretarias M unicipais deSaúde(SM S) dos municípios selecionados proveram as fontes de evidências, as quais variaram segundo a natureza do dado utilizado.

As fontes dados primários foram: gestor municipal, usuários do SUS e os profissionais de saúde das unidades de saúde - postos de saúde, unidade de PSF, centro de saúde, unidades mistas, hospitais municipais ou conveniados da zona urbana, existentes até o dia 31 de dezembro de 2002, considerando a vigência do PIACM. As unidades básicas de saúde da zona rural foram excluídas do estudo, devido às dificuldades operacionais, à distância e acesso.

Como fontes de dados secundários foram utilizadas: Sistema de Informação da M alária (SISM AL), Sistema de I nformação sobre M ortalidade (SIM ) eSistema de Informações H ospitalares do Sistema único de Saúde (SIH/SUS), dados do Departamento de Informação e Informática do Sistema Ú nico de Saúde (DATASUS), Instituto Brasileiro de Geografia e Estatística (IBGE), e do Instituto Nacional de Colonização e Reforma Agrária (INCRA).

Coleta de dados

As entrevistasforam real izadas de abril a agosto de 2004, nos locais de trabal ho de cada base de evidência, por cinco entrevistadores de nível superior. Estes entrevistadores foram treinados e os instrumentos de coleta de dados primários testa- 
dos em dois CASOS mais próximos da capital.

Entrevistou-se o gestor municipal, os profissionais de saúde de cada unidade - um médico, um enfermeiro da unidade, um bioquímico ou microscopista, dois agentes comunitários de saúde (PSF, PACS ou PASCAR) e dois agentes de saúde ambiental (ASA) -, que foram selecionados aleatoriamente. Também foi entrevistado o coordenador de endemias (ou profissional de controle de campo), um agente de campo que trabalha diretamente no controle da malária e dois usuários que estavam na unidade, selecionados al eatoriamente no momento da pesquisa. Quanto ao gestor, foi considerada a pessoa que estava exercen do o cargo de secretário municipal de saúde no momento da pesquisa.

A coleta de dados em cada CASO foi realizada observando-se o seguinte procedimento: a) primeiramente, entrevistou-se o secretário municipal de saúde (gestor), que recebeu uma carta de apresentação do entrevistador e explicação sobre a pesquisa, assinando então o termo de consentimento para real ização da pesquisa no município; b) posteriormente, os entrevistadores percorreram as unidades de saúde para entrevistar os profissionais de saúde e usuários; c) todos os entrevistados receberam uma carta de apresentação com explicação sobre a pesquisa e assinaram um termo de consentimento de participação.

Processamento e análise dos dados

0 estudo decaso incorporado exigiu duas etapas de análise: a interna e a cruzada dos casos. $\mathrm{Na}$ primeira, cada unidadedeanálise foi tratadacomo uma unidade abrangente em si mesma, e os dados foram analisados e triangulados dentro da integridade desse caso. A seguir, fez-se a análise cruzada, que permitiu desenvolver abstrações, comparando as unidades eidentificando quais os principais pontos comunsque contribuíram para um maior ou menor nível de implantação.

As análises cruzadas, no estudo de caso único incorporado, objetivaram reunir os diversos níveis deexplicação deum fenômeno. Analisaramse, dentro de um caso, as várias subunidades. Os vários níveis de análise acrescentaram oportunidades para aumentar o entendimento da implantação. Essa análise foi realizada no decurso da observação dos comportamentos e processos organizacionais em vários estágios.

Desta forma, os dados primários levantados nos municípios foram consolidados na matriz de avaliação, utilizando as tabelas do M icrosoft Excel do Windows 2000. Os dados dos profissio- nais de saúde que fizeram parte desta base de evidência foram consolidados na matriz e, posteriormente, realizada a média por critério/indicador de cada subdimensão, a qual correspondeu o valor observado desta base de evidência na matriz de análise. Em seguida, foram somadas as cinco bases de evidências e obteve-se a pontuação máxima observada de cada indicador por subdimensão e dimensão.

Com o total de pontos em relação ao valor esperado e observado, calculou-sea porcentagem, segundo a dimensão por cada CASO ${ }^{11}$. Sendo:

$$
\%=\sum_{i=1}^{n} \frac{Y(0)}{Y(E)} \times 100
$$

onde:

$Y(0)$ : é o valor observado da dimensão;

$Y(E)$ : é o valor esperado de cada dimensão.

Através do percentual e com os pontos de corte (escores) estabelecidos pelo consenso, classificou-se as dimensões em condição adequada, aceitável einsatisfatória. Foi consideradaadequada quando o percentual atingiu $75,0 \%$ ou mais da pontuação máxima; aceitável quando esteve entre 40,0 e 74,9\%; insatisfatório quando o percentual não alcançou $40,0 \%{ }^{12}$.

Após a classificação das dimensões, foram analisados os determinantes contextuais (externo, político e organizacional) que podem influenciar a VE no PIACM e seus efeitos. A implementação foi analisada a partir da VE no PIACM eindicadores de efeito.

\section{Resultados}

Os dados dos dezoito CASOS analisados foram consolidados na M atriz deJ ulgamento ou Análise apresentada na Tabela 1, com as mesmas dimensões e subdimensões que estruturam o modelo lógico de avaliação (M $L A)$ : contexto externo epolítico, contexto organizacional, implementação e efetividade.

Observou-se que a maioria dos CASOS apresentou a condição categorizada como aceitável ( 40 a 74,9\%) em todas as dimensões (Tabela 2).

$\mathrm{N}$ as dimensões contexto externo, político e organizacional, nenhum CASO obteve pontuação para ser classificado como adequado (e" 75,0\%); na dimensão implementação o CASO 11 e na efetividade o CASO 15 foram considerados como adequado. Dezessete CASO S tiveram classificação aceitável no contexto externo epolítico, variando o percentual de $45,7 \%$ a $74,6 \%$. Somente o CASO 9, dentre os dezoito CASOS, apresen- 
tou percentual menor que $40,0 \%$, sendo classificado, assim, como insatisfatório (Tabelas 1 e 2).

$\mathrm{N}$ a dimensão contexto organizacional, foram observados oito CASOS com situação insatisfatória e os demais CASOS com classificação aceitável (Tabelas 1 e 2).
$\mathrm{Na}$ dimensão implementação, observou-se uma melhora na classificação dos CASOS, pois haviam dezessete CASOS com situação aceitável e somente o CASO 11 foi classificado como adequado (Tabelas 1 e 2).

Tabela 1. Distribuição dos CASOS segundo pontuação em percentual das dimensões e sub-dimensões da M atriz de julgamento ou análise, M ato Grosso, 2005.

\begin{tabular}{|c|c|c|c|c|}
\hline \multirow[t]{2}{*}{ Dimensões e sub-dimensões } & \multirow[t]{2}{*}{$\begin{array}{l}\text { Ponto } \\
\text { Máx. }\end{array}$} & \multicolumn{3}{|c|}{$\begin{array}{l}\text { Pontuação em } \\
\text { percentual (\%) dos } \\
\text { CASOS estudados }\end{array}$} \\
\hline & & 1 & 2 & 3 \\
\hline 1. CONTEXTO EXTERNO E POLÍTICO & 810 & 70,5 & 48,8 & 45,9 \\
\hline 1.1 Condições socioeconômicas e ambientais & 210 & 52,4 & 45,2 & 45,2 \\
\hline 1.2 Projeto de governo e governabilidade & 180 & 70,3 & 66,8 & 41,3 \\
\hline 1.3 Capacidade de governo & 420 & 79,6 & 42,7 & 48,3 \\
\hline 2. CONTEXTO ORGANIZACIONAL & 815 & 37,8 & 49,9 & 35,2 \\
\hline 2.1 Planejamento e monitoramento & 265 & 15,1 & 81,4 & 44,5 \\
\hline 2.2 Capacitação de recursos humanos & 200 & 22,7 & 25,0 & 19,0 \\
\hline 2.3 Opinião do RH & 100 & 100,0 & 16,7 & 15,4 \\
\hline 2.4 Informação, Educação e Comunicação (IEC) e mobilização social & 250 & 49,0 & 49,5 & 46,3 \\
\hline 3. IM PLEM ENTAÇÃ O & 1.750 & 68,6 & 56,2 & 57,8 \\
\hline 3.1 Integralidade e Qualidade da Vigilância Epidemiológica e Ambiental & 425 & 75,3 & 61,8 & 58,2 \\
\hline 3.2 Integralidade e qualidade dos serviços de saúde & 825 & 71,9 & 53,5 & 59,2 \\
\hline 3.3 Implementação da infra-estrutura & 500 & 57,7 & 55,8 & 55,1 \\
\hline 4. EFETIVIDADE & 1.300 & 62,8 & 41,5 & 59,6 \\
\hline 4.1 Controle de riscos & 800 & 58,3 & 25,0 & 68,8 \\
\hline 4.2 Controle dos danos & 400 & 100,0 & 75,0 & 50,0 \\
\hline 4.3 Satisfação dos usuários & 100 & 100,0 & 40,0 & 25,0 \\
\hline
\end{tabular}

\begin{tabular}{rrrrrrrrrrrrrrc}
\hline 4 & 5 & 6 & 7 & 8 & 9 & 10 & 11 & 12 & 13 & 14 & 15 & 16 & 17 & 18 \\
65,9 & 49,6 & 55,6 & 56,5 & 59,8 & 39,8 & 66,2 & 70,4 & 61,3 & 45,7 & 71,7 & 56,4 & 56,7 & 51,6 & 74,6 \\
52,4 & 40,5 & 57,1 & 50,0 & 50,0 & 38,1 & 42,9 & 47,6 & 64,3 & 50,0 & 47,6 & 38,1 & 52,4 & 54,8 & 57,1 \\
67,7 & 42,3 & 57,9 & 30,4 & 56,0 & 20,2 & 55,6 & 70,5 & 63,1 & 50,2 & 79,2 & 66,2 & 32,7 & 41,7 & 89,9 \\
71,9 & 57,4 & 53,8 & 71,0 & 66,2 & 48,9 & 82,5 & 81,7 & 59,0 & 41,6 & 80,7 & 61,3 & 69,2 & 54,3 & 76,7 \\
56,6 & 38,8 & 54,0 & 55,8 & 61,1 & 44,2 & 36,5 & 62,9 & 28,6 & 61,1 & 39,3 & 44,9 & 37,7 & 20,8 & 47,5 \\
81,3 & 7,3 & 90,3 & 27,9 & 78,9 & 23,8 & 29,2 & 88,7 & 10,0 & 65,7 & 9,4 & 76,1 & 14,9 & 13,4 & 11,6 \\
22,0 & 40,0 & 33,6 & 79,3 & 45,8 & 23,9 & 21,9 & 18,7 & 10,8 & 49,1 & 41,7 & 26,2 & 40,8 & 7,1 & 63,5 \\
58,0 & 88,8 & 37,2 & 42,1 & 19,0 & 100,0 & 42,6 & 80,0 & 66,5 & 25,0 & 48,0 & 18,1 & 62,5 & 16,7 & 62,5 \\
57,4 & 51,2 & 38,5 & 71,9 & 71,3 & 59,8 & 53,5 & 63,9 & 47,2 & 80,4 & 65,4 & 37,4 & 49,4 & 41,3 & 66,7 \\
57,0 & 56,8 & 63,0 & 50,2 & 67,4 & 51,6 & 64,9 & 79,8 & 53,7 & 61,1 & 68,6 & 55,6 & 61,7 & 56,1 & 65,4 \\
65,2 & 65,4 & 56,9 & 58,8 & 77,5 & 38,1 & 66,9 & 91,5 & 67,4 & 44,3 & 81,8 & 59,4 & 62,8 & 66,7 & 69,6 \\
58,1 & 62,8 & 69,4 & 45,1 & 74,6 & 67,4 & 70,7 & 77,5 & 47,6 & 64,2 & 67,0 & 66,8 & 60,3 & 54,3 & 75,5 \\
48,2 & 39,8 & 57,6 & 51,1 & 46,8 & 36,9 & 53,6 & 73,7 & 52,0 & 70,2 & 60,0 & 33,7 & 63,0 & 50,2 & 45,3 \\
69,2 & 53,9 & 50,0 & 57,7 & 70,1 & 73,1 & 53,9 & 48,1 & 66,8 & 34,6 & 56,4 & 82,7 & 73,7 & 53,9 & 65,4 \\
50,0 & 37,5 & 25,0 & 50,0 & 56,3 & 68,8 & 37,5 & 25,0 & 50,0 & 25,0 & 50,0 & 75,0 & 66,7 & 37,5 & 50,0 \\
100,0 & 75,0 & 100,0 & 75,0 & 100,0 & 87,5 & 100,0 & 87,5 & 100,0 & 50,0 & 75,0 & 100,0 & 100,0 & 100,0 & 87,5 \\
100,0 & 100,0 & 49,8 & 50,0 & 61,5 & 49,8 & 0,0 & 75,0 & 68,1 & 49,8 & 33,2 & 75,0 & 25,0 & 0,0 & 100,0 \\
\hline
\end{tabular}

Parâmetros para avaliação da pontuação das dimensões e subdimensões: A dequado $\geq 75,0 \%$; Aceitável 40,0 a 74,9\%; Insatisfatório $<40,0 \%$. 
Tabela 2. N úmero de CASOS segundo dimensões sub-dimensões conforme os parâmetros adotados.

\begin{tabular}{|c|c|c|c|c|}
\hline \multirow[t]{2}{*}{ Dimensões e sub-dimensões } & \multicolumn{3}{|c|}{ Número de CASOS com parâmetros } & \multirow{2}{*}{$\begin{array}{l}\text { Total de } \\
\text { CASOS }\end{array}$} \\
\hline & $\begin{array}{l}\text { Adequado } \\
(\geq 75,0 \%)\end{array}$ & $\begin{array}{c}\text { Aceitável } \\
(40,0 \text { a } 74,9 \%)\end{array}$ & $\begin{array}{c}\text { Insatisfatório } \\
(>40,0 \%)\end{array}$ & \\
\hline 1. Contexto externo e político & - & 17 & 1 & 18 \\
\hline 1.1 Condições socioeconômicas e ambientais & - & 16 & 2 & 18 \\
\hline 1.2 Projeto de governo e governabilidade & 2 & 13 & 3 & 18 \\
\hline 1.3 Capacidade de governo & 5 & 13 & - & 18 \\
\hline 2. Contexto organizacional & - & 10 & 8 & 18 \\
\hline 2.1 Planejamento e monitoramento & 6 & 2 & 10 & 18 \\
\hline 2.2 Capacitação de recursos humanos (RH) & 1 & 6 & 11 & 18 \\
\hline 2.3 Opinião do RH & 4 & 7 & 7 & 18 \\
\hline 2.4 Informação, educação e comunicação (IEC) e mobilização social & 1 & 15 & 2 & 18 \\
\hline 3. Implementação & 1 & 17 & - & 18 \\
\hline 3.1 Integralidade e qualidade da vigilância epidemiológica e ambiental & 4 & 13 & 1 & 18 \\
\hline 3.2 Integralidade e qualidade dos serviços de saúde & 2 & 16 & - & 18 \\
\hline 3.3 Implementação da infra-estrutura & - & 15 & 3 & 18 \\
\hline 4. Efetividade & 1 & 16 & 1 & 18 \\
\hline 4.1 Controle de riscos & 1 & 10 & 7 & 18 \\
\hline 4.2 Controle dos danos & 16 & 2 & - & 18 \\
\hline 4.3 Satisfação dos usuários & 6 & 7 & 5 & 18 \\
\hline
\end{tabular}

$\mathrm{Na}$ dimensão efetividade das ações de controle da malária, dezesseis casos foram classificados como aceitável e apenas um (CASO 13), como insatisfatório (Tabelas 1 e 2).

Do total de dezoito municípios, quinze $(83,3 \%)$ apresentaram as dimensões implementação e efetividade em condições aceitáveis, mas as demais dimensões de contexto apresentaram variação entre aceitável e/ou insatisfatória.

Nas três subdimensões do contexto externo e político, mais da metade dos CASOS concentraram-se na condição aceitável. Vale ressaltar que na subdimensão capacidade de governo, cinco CASO S classificaram-se na condição de adequada, mas em contrapartida, na subdimensão projeto de governo e governabilidade, três CASOS foram considerados como insatisfatórios.

No contexto organizacional, os CASOS $10 \mathrm{e}$ 11 foram classificados como insatisfatórios nas subdimensões planejamento e monitoramento, e capacitação de recursos humanos, respectivamente. Quanto à subdimensão opinião dos Recursos Humanos, IEC e mobilização social, a maioria dos CASOS se concentraram na condição aceitável. Ressalta-se apenas que a opinião do RH teve quatro CASOS adequados, sete aceitáveis e sete insatisfatórios.

As subdimensões da implementação também tiveram mais de $70 \%$ dos CASOS concentrados na condição aceitável. D estaca-sea subdimen são integral idade equalidade da vigilância epidemiológica, na qual quatro CASOS encontram-se na condição adequada.

Os indicadores de efetividade das ações de controle também foram bem classificados. No controle de risco, dez CASOS se classificaram como aceitáveis, enquanto sete foram insatisfatórios. Já no controle de danos, dezesseis CASOS foram adequados. $\mathrm{Na}$ última subdimensão da efetividade - satisfação do usuário - seteCASOS classificaram-se como adequados (Tabela 2).

\section{Discussão}

Por tratar-se de uma investigação de fenômenos contemporâneos contextual izados na vida real, $\mathrm{e}$ como os limites entre o fenômeno e o contexto não são claramente definidos, o tipo de estudo mais recomendado foi o estudo de caso ${ }^{10}$. Esta abordagem permite diferenciar as características do meio e os efeitos produzidos pela intervenção de uma implantação ou implementação ${ }^{13}$, mediante descrições e análises intensivas de uma única unidade (CASO) ou sistema circunscritos ${ }^{17}$.

Para garantir a validade interna, optou-seem utilizar o modelo de avaliação rápida (REM ), que atende a recomendação para estudo de casos e a 
triangulação de dados de forma sistemática e objetiva, auxiliando assim a verificação contínua da confiabilidade, validadeeinterpretação da informação coletada ${ }^{14,15}$.

Neste estudo, nenhum CASO foi considerado como adequado na dimensão contexto externo e político. 0 fator que mais contribuiu para essa classificação foram os assentamentos rurais oficiais existentes em dezessete dos dezoito CASOS, pois a malária em M ato Grosso, nas últimas décadas, concentrou-se principalmente em assentamentos rurais e garimpos. Segundo Sawyer ${ }^{18}$, em novos assentamentos rurais localizados em florestas úmidas, ocorreram epidemias de malária na fase inicial de sua implantação, especialmente quando estes foram desenvolvidos deforma desorganizada ou insuficientemente dirigidos. A el evada prevalência é atribuída a vários fatores, como: a falta de organização de estrutura sanitária, condições precárias de habitação e desmatamento. Também Barata ${ }^{7}$ ressaltava que os casos de malária na região amazônica eram relacionados com a alta mobilidade, por razões de ocupação da terra e exploração das riquezas (minerais e vegetais).

Estecontexto relacionado ao processo de ocupação recente é reforçado pelo tempo de criação dos municípios do estado de M ato Grosso, prioritários do PIACM , sendo que dezesseis deles foram criados nas décadas de 1980 e 1990.

Os CASOS apresentavam, em 2004, IDH variando de 0,500 a 0,799, sendo classificados como médio desenvolvimento ${ }^{19}$. Em relação ao saneamento básico, segundo dados do Censo de 2000 ${ }^{20}$, o percentual de domicílios quepossuíam abastecimento de água procedente da rede geral encontrava-se menor que $62,0 \%$, bem abaixo da média nacional $(77,8 \%)$, enquanto que a cobertura de serviço de coleta de lixo era maior, com $80 \%$ dos CASOS beneficiados com o serviço. $\mathrm{N}$ este aspecto, a disponibilidade eacesso aos serviços básicos de infra-estrutura sanitária forneceram indicativos importantes a respei to da qualidade de vida da população dos CASOS.

Por outro lado, o projeto de governo egovernabilidade, ea capacidade de governo foram classificados como aceitáveis em $72,2 \%$ dos CASOS. Todos os CASOS receberam mensalmente repasses financeiros para gestão do SUS municipal, inclusiveo Teto Financei ro deVigilância em Saúde $(T F V S)^{21}$. Este repasse regular e estável, mesmo em pequenas proporções, assegura aos municípios as condições necessárias para a elaboração do planejamento e execução das ações, conforme $\mathrm{H}$ eiman et al. ${ }^{22}$. Se, no entanto, o repasse de recursos caracteriza-se como facilitador da implementação do programa de controle da malária, observou-se que muitos profissionais e gestores desconheciam o repasse para os municípios do TFVS (antigo TFECD - Teto Financeiro de Epidemiologia e Controle de Doenças).

0 desconhecimento do repasseespecífico para a vigilância em saúde pode ser explicado pela elevada rotatividade dos gestores municipais nas SM S. O pequeno tempo de atuação ea oscilação política comprometem o projeto de governo e governabilidade, que por sua vez podem influenciar a gestão do município e, conseqüentemente, os programas de saúde em nível local.

A rotatividade não esteve restrita aos gestores; identificou-se também elevada rotatividade entre os profissionais de saúde, especialmente os da equipe de Programa Saúde da Família (PSF), o que podeinfluenciar negativamenteo processo de descentralização das ações de controle da malária. Para Gil${ }^{23}$, a instabilidade do profissional nos serviços básicos do SUS é decorrente da fragilidade dos vínculos empregatícios das equipes, que sofrem influência da gestão municipal. Verificou-se que a estabilidade do profissional de saúde pode significar um ganho aos serviços de saúde, pois há uma consolidação e acúmulo de conhecimento e habilidade do profissional.

Outro indicador importante foi a capacitação dos Recursos Humanos, da subdimensão capacidade de governo. As capacitações foram esporádicas e, em cada setor da saúde (central e/ ou local), existe o trabalho fragmentado, ou seja, profissionais de atenção básica, vigilância ambiental, epidemiológica e assistência trabalham de forma desarticuladas.

No contexto organizacional, observou-se $44,4 \%$ dos CASO $S$ com classificação insatisfatória, o que pode ser de certa forma uma influência do contexto externo epolítico decadaCASO. Vale ressaltar que os principais indicadores que contribuíram com esta condição foram: precário planejamento das ações; capacitações esporádicas e fragmentadas; atividades educativas pontuais; material educativo e ações educati vas quase insuficientes, e uma baixa implementação do PSF duranteo período de 1999 a 2002 nos municípios prioritários. Soma-se a isso, uma insatisfação generalizada, referida pelos profissionais de saúde.

Alguns estudos relatam que o contexto organizacional dos municípios ainda é um grande desafio para o SUS. Drumond Jr. ${ }^{24}$ destacava a dificuldade de capacitar os profissionais do SUS para aprimorar suas práticas nos diferentes ní- 
veis. Já Carvalho \& M arzocchi ${ }^{25}$ constataram que os profissionais de saúde possuem acúmulo de funções e condições de trabalho precárias que geram insatisfações. Gil ${ }^{23}$ descreveu que os profissionais de saúde não estão aptos a desempenhar funções cotidianas de atenção básica ao indivíduo eà família, eisto se deveà supervalorização das práticas da medicina curativa, especializada, de procedimentos tecnológicos e medicalizada que os mesmos recebem na sua formação, que por sua vez podem levar à fragmentação das ações de saúde. Dias ${ }^{6}$ afirmou, em seu estudo, quea organização ebom funcionamento dos sistemas de saúde constituem peças fundamentais à vigilância da malária.

Ressalta-seque o contexto organizacional não foi considerado adequado em nenhum CASO, mas teve dez dos dezoito CASOS classificados como aceitáveis. Alguns indicadores que contribuíram para esta condição foram: mapeamento atualizado dos principais focos da malária no município; ações de controle voltadas para as populações de assentamento, garimpo e de áreas de extrativismo; e o nível de informação do usuário sobre a malária, principalmentesobreossintomas da doença. De acordo com Dias ${ }^{6}, 0$ conhecimento da população sobre a malária contribui para a prevenção individual, para 0 acesso aos serviços, e para o diagnóstico e tratamento correto da doença.

Contudo, constatou-se que os usuários das unidades dos CASOS não sabiam identificar a forma correta de infecção e de prevenção. Isto apesar de existir protocolos relativos ao controle da malária, que preconizam como realizar as ações deeducação em saúdeemobilização social, para informar todos os setores da sociedade quanto à forma deprevenção, deinfecção, eacerca dos principais sintomas da doença. Esta constatação pode limitar as possibilidades de adoção de medidas preventivas, tanto individuais quanto coletivas.

A implementação da vigilância epidemiológica foi adequada em apenas um CASO e aceitável nos demais. A vigilância epidemiológica e ambiental teve $72,2 \%$ dos CASOS em condições aceitáveis, restrita principal mente à coleta de dados, através de notificações e investigações, e à alimentação dos sistemas de informação. As outras atividades específicas da vigilância, principalmente as preconizadas pelo Guia de Vigilância Epidemiológica ${ }^{26}$, foram pouco adotadas pelos CASOS. Carvalho \& M arzocchi ${ }^{25}$ eCerqueira et al. ${ }^{27}$ enfatizavam nas suas avaliações que as atividades de vigilância epidemiológica sempre tiveram um caráter localizado, freqüentemente reduzindo à implantação de medidas de controle. Por outro lado, Barata7 recomendava para os municípios da Amazônia Legal a manutenção de sistema devigilância efetivo, com busca ativa constante dos casos suspeitos de malária. A atividade de busca ativa dos casos de malária foi prática esporádica pela atenção básica, contrariando o preconizado pelo plano anterior (PIACM $)^{16}$. Bodstein ${ }^{28}$ apontava que o programa de atenção básica ainda não se encontra sintonizado com os determinantes e condicionantes dos agravos no município.

A integralidade e a qualidade dos serviços de saúde também se mostraram em condição aceitável em 88,9\% dos CASOS; porém, o que mais se diferenciou do preconizado pelo PIACM foi a integralidade das ações de controle nas equipes de atenção básica. 0 diagnóstico e tratamento ocorriam de forma centralizada em apenas uma unidade de saúde, o que reduz a prática deacompanhamento do tratamento pela lâmina de verificação de cura (LVC). Algumas dessas divergências encontradas podem ser atribuídas ao processo de municipalização e descentralização das ações de endemias para as secretarias estaduais e municipais. A maioria dos municípios não estava preparada para assumir as ações de controle da malária que, anteriormente, era executado de forma centralizada na esfera federal, tanto pela SU CAM (1970-1989), como pela FNS (19901998) e FUNASA (1999-2000) 29-31. E, ainda, o cenário de heterogeneidade do PSF nos municípios possivel mente reduz a acessibilidade da população aos serviços de saúde, no que tange às ações de controle de malária.

Vale ressaltar que as visitas domiciliares realizadas pelos agentes da aten ção básica e agentes da saúde ambiental nos CASOS contribuíram para melhorar o acesso aos serviços de saúde. Esta prática, abordada por Senna ${ }^{32}$, representa importantemeio deinclusão daquel es que, habitualmente, encontram-se excluídos do sistema de saúde.

Outro fator positivo da integralidadee qualidade da atenção básica nos CASOS foi a incorporação do Programa de Apoio à Saúde Comunitária nos Assentamentos Rurais em $2001^{33}$, que propiciou a implementação das ações de vigilância, de atenção básica e de controle da malária nos assentamentos rurais legalizados pelo IN CRA ou INTERM AT.

0 ponto crítico da vigilância ainda é a infraestrutura, também citada por Cerqueira et al. ${ }^{27}$. Dos dezoito CASOS, a infra-estrutura em condi- 
ção aceitável ocorreu em 83,3\%; porém, muitos dos equipamentos, veículos e motocicletas repassados pelo PIACM ${ }^{8,16}$, em 2001, estavam sem condições de executar as ações de controle. A maioria dos municípios não possui local apropriado para armazenar inseticida e os equipamentos de borrifação. Quanto aos equipamentos de informática, estes estavam disponíveis apenas para utilização dossistemas informatizados, eem condições adequadas, 0 que é a realidade em muitos municípios, onde o acesso ao computador e à Internet são restritos, possivelmente, dificultando as atividades de análise dos dados coletados dos sistemas de informação em saúde.

Em relação à efetividade das ações de controle da malária, observou-se que $88,8 \%$ dos CASOS tiveram condições aceitáveis, sendo que as condições adequadas e insatisfatórias obtiveram, cada uma, 5,6\%. Os indicadores que mais se destacaram foram os de controle de danos com $88,8 \%$ dos CASOS com condição adequada. Já os indicadores de risco e a satisfação do usuário tiveram $5,6 \%$ e $33,3 \%$ dos CASOS com esta condição, respectivamente.

0 controle de risco possivelmente foi influenciado por algumas ações de vigilância e atenção básica, o que sinaliza que as ações de vigilância e controle devem ser executadas em todas as unidades de saúde. Sabe-se que isto ainda é um desafio. Cerqueira et al. ${ }^{27}$ destacavam que os profissionais de saúde das unidades básicas de saúde não incorporam a prática da vigilância epidemiológica em suas atividades, e muitos se limitam somente a notificar as doenças. Dias ${ }^{6}$ também reforçou a questão da organização e bom funcionamento dos serviços de saúde, pois constituem peças fundamentais à vigilância da malária.

Já os indicadores de controle de danos, como a mortalidade e a incidência parasitária anual, tiveram uma redução na maioria dos CASOS. Isso se deve, possivelmente, ao contexto externo dos CASOS, uma vez que a malária tem forte relação com o meio. Barbieri ${ }^{34}$ destacava que 0 equilíbrio ecológico das populações de mosquitos e do homem deve ser estável, e as condições naturais do meio pouco modificadas, para que a incidência da doença em humanos se mantenha em níveis estáveis. Porém, o desequilíbrio ocorre com a entrada de grandes contingentes humanos no meio, modificando 0 ambiente natural e alterando as características reprodutivas e 0 habitat dos vetores. A pesar da dimensão de contexto externo influenciar naintervenção do PIACM , esses CASOS já possuíam certa estabilidade no fluxo de pessoas, pois todos tinham mais de uma década deestruturação. Desta forma, pressupõese queas outras dimensões tiveram influência na incidência da doença.

Segundo Campbell et al ${ }^{35}$, os principais efeitos da qualidade da atenção à saúde dos indivíduos estão relacionados ao acesso, evidenciados pelo estado de saúde e satisfação do usuário. N esse estudo, os usuários dos serviços de saúde dos CASOS se mostraram satisfeitos com a atenção à saúde, indicando segundo a satisfação dos usuários, a existência de acesso e qualidade da atenção.

Dessa forma, ao considerar a malária em $M$ ato Grosso como uma endemia localizada e focal, os critérios para avaliação do programa, ao abordar dimensões abrangentes do âmbito municipal, possibilitaram considerar as peculiaridades eespecificidades do local queinfluenciam fortemente o resultado de uma intervenção.

\section{Conclusão}

Por fim, cada município necessita fortalecer os seus serviços de saúde, realizar integração entre as equipes e adotar estratégias de ação articuladas, a fim demanter estes indicadores de controle de danos em níveis aceitáveis, considerando as especificidades locais.

As principais conclusões foram: a) o contexto externo e político influenciaram as demais dimensões analisadas; b) o contexto organizacional, dos serviços de saúde, também exerceu influência na implementação e efetividade das ações de controle da malária, destacando a desarticulação entre as ações de vigilância e controle e de atenção básica.

Outro aspecto a se destacar foi a metodologia do estudo, que através de análises qualitativas comparativas, permitiu avaliar o processo de implementação das ações de controle da malária (PIACM) em dezoito municípios do estado de M ato Grosso. Possibilitou obter informações contextualizadas efocalizadas em nível local que, na avaliação tradicional, não seriam identificadas, e analisar as influências e articulações entre as dimensões envolvidas na implementação do programa.

Esteestudo reforça a importância dessa abordagem metodológica para o planejamento e a gestão de serviços de saúde, contribuindo para articulação e fortalecimento das ações básicas e desenvolvimento efetivo da vigilância em saúde. 


\section{Colaboradores}

ND Galvão participou da coleta dos dados, realizou as análises e escreveu o artigo; EM Yokoo, M Atanaka-Santos e $S \mathrm{~N}$ atal participaram das análises de dados, da redação e correção do artigo.

\section{Agradecimentos}

A Zulmira M aria deA raújo H artz, Elizabeth M oreira dos Santos, J oão H enrique Gurtler Scatena e ao Projeto do Programa Norte de Pesquisa e Pós-Graduação (PNOPG/CNPq) que foi financiado pelo CN Pq.

\section{Referências}

1. World Health Organization. Why tackle malaria? M alaria-At-A Glance Roll Back M alaria. Geneva; 2001. [acessado 2001 Ago 10]. Disponível em: http:/ /www.who.org/tdr

2. Fundação Nacional de Saúde. Situação da prevenção e controle de doenças de notificação compulsória e endêmicas no Brasil. Vigilância Epidemiológica. 2001. [acessado 2001 Nov 10]. Disponível em: http:/ /www.funasa.gov.br/

3. Marques AC, Pinheiro EA, Sousa AG. Um Estudo sobre a dispersão de casos de malária no Brasil. Rev Bras Malariol Doen Trop 1986; 38:51-75.

4. Brasil. M inistério da Saúde. Manual de terapêutica da malária. Brasília: Ministério da Saúde; 2001.

5. Brasil. Ministério da Saúde. Secretaria de Vigilância em Saúde. Coordenação Geral do Programa Nacional de Controle da Malária. Plano de Intensificação das Ações de Controle da Malária na Amazônia Legal (PIACM) período julho de 2000 a dezembro de 2002: relatório de gestão. Brasília; M inistério da Saúde; 2003.

6. Dias JCP. Participação, descentralização e controle de endemias no Brasil. In: Barata RB, organizador. Doenças endêmicas: abordagens sociais culturais e comportamentais. Rio de Janeiro: Fiocruz; 2000. p. 269-277.

7. Barata RCB. Malária no Brasil: panorama epidemiológico na última década. Cad Saúde Pública 1995; 11(1):128-136.

8. Brasil. M inistério da Saúde. Plano Nacional de Controle da M alária. Brasília: M inistério da Saúde; 2003.

9. Braz RM. Detecção precoce de epidemias de malária no Brasil: uma proposta de automação. Epidemiol Serv Saúde 2006; 15(2):21-33.

10. Yin RK. Estudo de caso: planejamento e métodos. $2^{\text {a }}$ ed. Porto Alegre: Bookman; 2001.

11. Natal S, Hartz Z, Santos EM, Cruz M M, Patroclo MA. Avaliação dos programas de controle da malária, dengue, tuberculose e hanseníase: estudo de casos municipais/estaduais na Amazônia legal [relatório final]. Rio de Janeiro: DEN SP/EN SP/Fiocruz; 2004.

12. Natal S, Penna M LF, Hartz Z, Santos EM, Sabroza P. Avaliação do programa de controle da tuberculose: estudos de casos municipais/estaduais na Amazônia Legal. Bol Pneumol Sanit 2004; 12(2):91-110.

13. Hartz ZA. Avaliação em Saúde: dos modelos conceituais à prática na análise de implantação de programas. Rio de Janeiro: Fiocruz; 1997.

14. Stimson GV, Donoghoe MC, Fitch C, Rhodes TJ, Ball A, Weiler G, editors. Rapid Assessment and Response Technical Guide. Version 1.0. Geneva: WHO; 2003.

15. World Health Organization. Rapid evaluation method: guidelines for maternal and child health, family planning and other health services. Geneva: WHO; 1993.

16. Brasil. M inistério da Saúde. Plano Nacional de Intensificação das A ções de controle da M alária na Amazônia legal. Brasília: M inistério da Saúde; 2000.

17. Merriam SB. Case study research in education: a qualitative approach. San Francisco, CA: J ossey-Bass; 1988.

18. Sawyer DR. Malaria and the environment. Brasília: ISPN; 1992. 
19. Secretaria de Estado de Planejamento de M ato Grosso. Boletim sócio-economico-demográfico dos municípios mato-grossenses. Cuiabá: Secretaria de Estado de Planejamento de M ato Grosso; 2004.

20. Instituto Brasileiro de Geografia e Estatística. Anuário estatístico do Brasil. Rio de Janeiro: IBGE; 2000.

21. Fundo Nacional de Saúde. Consulta de pagamentos - Fundo a Fundo. 2004. [acessado 2004 Ago 20]. Disponível em: http://www.fns.saude.gov.br/

22. Heimann LS, Cortizo CT, Castro IEN, Kayano J, Rocha JL, N ascimento PR, Boaretto RC, Pessoto UC, Junqueira Virginia. Descentralização do sistema único de saúde: trilhando a autonomia municipal. São Paulo: Sobravime; 2000. p. 99-111.

23. Gil CRR. Formação de recursos humanos em saúde da família: paradoxos e perspectivas. Cad Saúde Pública 2005; 21(2):490-498.

24. Drumond Jr M. Epidemiologia nos municípios muita além das normas. São Paulo: Hucitec; 2003.

25. Carvalho MS, Marzocchi KBF. Avaliação da prática de vigilância epidemiológica nos serviços públicos de saúde no Brasil. Rev. Saúde Pública 1992; 26(2):66-74.

26. Brasil. M inistério da Saúde. Guia de vigilância epide miológica. 6a ed. Brasília: Ministério da Saúde; 2005.

27. Cerqueira EM, Santos DG, Assis M MA. Vigilância epidemiológica no processo de municipalização do sistema de saúde em Feira de Santana-BA. Epidemiol Serv Saúde 2003; 12(4):213-223.

28. Bodstein R. Atenção básica na agenda da saúde. Cienc Saude Colet 2002; 7(3):401-412.

29. Barata RCB. M alária e seu controle. São Paulo: Hucitec; 1998.

30. Tauil PL. Avaliação de uma nova estratégia de controle da malária na Amazônia Brasileira [tese]. Brasília (DF): Universidade de Brasília; 2002.

31. Loiola CCP, Silva CJM, Tauil PL. Controle da malária no Brasil: 1965 a 2001. Rev Panam Salud Publica 2002; 11(4):235-244

32. Senna MCM. Eqüidade e política de saúde: algumas reflexões sobre o Programa Saúde da Família Cad Saúde Pública 2002; 18(Supl):203-211.

33. Secretaria de Estado de Saúde de M ato Grosso. A regionalização da saúde em $M$ ato Grosso: em busca da integralidade da atenção. Cuiabá: SES; 2002.

34. Barbieri AF. U so antrópico da terra e malária no Norte de M ato Grosso, 1992 a 1995 [dissertação]. Belo Horizonte (MG): Universidade Federal de Minas Gerais; 2000

35. Campbell SM, Roland M O, Buetow SA. Defining quality of care. Social Science and M edicine 2000 51:1611-1625.

36. Denis JL, Champagne F. Análise da implantação. In: Hartz ZM A, organizadora. Avaliação em saúde: dos modelos conceituais à prática na análise da implantação de programas. Rio de Janeiro: Fiocruz 1997. p. 49-88.

Artigo apresentado em 17/08/2006

Aprovado em 15/08/2007

Versão final apresentada em 21/08/2007 